PROCEEDINGS OF THE

AMERICAN MATHEMATICAL SOCIETY

Volume 132, Number 4, Pages 987-996

S 0002-9939(03)07143-0

Article electronically published on July 17, 2003

\title{
HECKE ALGEBRAS FOR THE BASIC CHARACTERS OF THE UNITRIANGULAR GROUP
}

\author{
CARLOS A. M. ANDRÉ
}

(Communicated by Stephen D. Smith)

\begin{abstract}
Let $U_{n}(q)$ denote the unitriangular group of degree $n$ over the finite field with $q$ elements. In a previous paper we obtained a decomposition of the regular character of $U_{n}(q)$ as an orthogonal sum of basic characters. In this paper, we study the irreducible constituents of an arbitrary basic character $\xi_{\mathcal{D}}(\varphi)$ of $U_{n}(q)$. We prove that $\xi_{\mathcal{D}}(\varphi)$ is induced from a linear character of an algebra subgroup of $U_{n}(q)$, and we use the Hecke algebra associated with this linear character to describe the irreducible constituents of $\xi_{\mathcal{D}}(\varphi)$ as characters induced from an algebra subgroup of $U_{n}(q)$. Finally, we identify a special irreducible constituent of $\xi_{\mathcal{D}}(\varphi)$, which is also induced from a linear character of an algebra subgroup. In particular, we extend a previous result (proved under the assumption $p \geq n$ where $p$ is the characteristic of the field) that gives a necessary and sufficient condition for $\xi_{\mathcal{D}}(\varphi)$ to have a unique irreducible constituent.
\end{abstract}

Let $p$ be a prime number, let $q=p^{e}(e \geq 1)$ be a power of $p$, and let $\mathbb{F}_{q}$ denote the finite field with $q$ elements. Throughout the paper, we will denote by $U$ the the unitriangular group $U_{n}(q)$ of degree $n$ over $\mathbb{F}_{q}$; by definition, $U$ consists of all unipotent uppertriangular $n \times n$ matrices with coefficients in $\mathbb{F}_{q}$. We clearly have $U=1+\mathfrak{u}$ where $\mathfrak{u}=\mathfrak{u}_{n}(q)$ is the $\mathbb{F}_{q}$-space consisting of all nilpotent uppertriangular $n \times n$ matrices over $\mathbb{F}_{q}$; in particular, the $p$-group $U$ is an $\mathbb{F}_{q}$-algebra group (in the sense of [5]; see also [4). Moreover, let $\mathfrak{u}^{*}$ denote the dual $\mathbb{F}_{q}$-space of $\mathfrak{u}$.

For simplicity, we write $\Phi=\{(i, j): 1 \leq i<j \leq n\}$ and we refer to an element of $\Phi$ as a root. For any $(i, j) \in \Phi$, let $e_{i j} \in \mathfrak{u}_{n}(q)$ be the $n \times n$ matrix $e_{i j}=$ $\left(\delta_{r i} \delta_{s j}\right)_{1 \leq r, s \leq n}$ where $\delta$ denotes the usual Kronecker symbol. Then, $\left(e_{i j}:(i, j) \in \Phi\right)$ is an $\mathbb{F}_{q}$-basis of $\mathfrak{u}$ to which we will refer as the standard basis of $\mathfrak{u}$. On the other hand, for any $(i, j) \in \Phi$, let $e_{i j}^{*} \in \mathfrak{u}^{*}$ be defined by $e_{i j}^{*}(a)=a_{i j}$ for all $a \in \mathfrak{u}$ (given a matrix $x$, we will denote by $x_{i j}$ the $(i, j)$-th coefficient of $\left.x\right)$. Then, $\left(e_{i j}^{*}:(i, j) \in \Phi\right)$ is an $\mathbb{F}_{q}$-basis of $\mathfrak{u}^{*}$, dual to the standard basis of $\mathfrak{u}$.

Let $\psi: \mathbb{F}_{q}^{+} \rightarrow \mathbb{C}$ be an arbitrary nontrivial character of the additive group $\mathbb{F}_{q}^{+}$ of $\mathbb{F}_{q}$ (this character will be kept fixed throughout the paper) and, for any $f \in \mathfrak{u}^{*}$, let $\psi_{f}: \mathfrak{u} \rightarrow \mathbb{C}$ be the function defined by $\psi_{f}(a)=\psi(f(a))$ for all $a \in \mathfrak{u}$. It is clear that this function is a linear character of the additive group $\mathfrak{u}^{+}$of $\mathfrak{u}$ and that the mapping $f \mapsto \psi_{f}$ defines a one-to-one correspondence between $\mathfrak{u}^{*}$ and the set of all

Received by the editors September 26, 2002 and, in revised form, December 3, 2002.

2000 Mathematics Subject Classification. Primary 20C15; Secondary $20 \mathrm{G} 40$.

Key words and phrases. Unitriangular group, irreducible character, basic character. 
irreducible characters of $\mathfrak{u}^{+}$. (Throughout the article, all characters are taken over the complex field.)

The group $U$ acts on $\mathfrak{u}^{*}$ via the coadjoint representation: for any $x \in U$ and any $f \in \mathfrak{u}^{*}$, we define the linear map $x \cdot f \in \mathfrak{u}^{*}$ by $(x \cdot f)(a)=f\left(x^{-1} a x\right)$ for all $a \in \mathfrak{u}$. Let $\mathcal{O} \subseteq \mathfrak{u}^{*}$ be an arbitrary $U$-orbit. By [2, Lemma 1], we know that the cardinality $|\mathcal{O}|$ of $\mathcal{O}$ is a power of $q^{2}$. Let $\phi_{\mathcal{O}}: U \rightarrow \mathbb{C}$ be the class function defined by

$$
\phi_{\mathcal{O}}(1+a)=\frac{1}{\sqrt{|\mathcal{O}|}} \sum_{f \in \mathcal{O}} \psi(f(a))
$$

for all $a \in \mathfrak{u}$. In general, $\phi_{\mathcal{O}}$ is not a character (see [6]). However, there are some examples where they are, in fact, irreducible characters of $U$. A particular family consists of the elementary characters of $U$, which are defined as follows. Let $(i, j) \in \Phi$ be any root and let $\alpha \in \mathbb{F}_{q}$ be any nonzero element. (Throughout the paper, we will denote by $\mathbb{F}_{q}^{\times}$the subset of $\mathbb{F}_{q}$ consisting of all nonzero elements.) Let $\mathcal{O}_{i j}^{*}(\alpha) \subseteq \mathfrak{u}^{*}$ be the $U$-orbit that contains the element $\alpha e_{i j}^{*} \in \mathfrak{u}^{*}$, and let $\xi_{i j}(\alpha)$ denote the class function $\phi_{\mathcal{O}_{i j}^{*}(\alpha)}$ that corresponds to $\mathcal{O}_{i j}^{*}(\alpha)$. By [2, Lemma 2], we know that this class function is, in fact, an irreducible character of $U$. We will refer to $\xi_{i j}(\alpha)$ as the $(i, j)$-th elementary character of $U$ associated with $\alpha$.

Now, a subset $\mathcal{D} \subseteq \Phi$ is called a basic subset if $|\mathcal{D} \cap\{(i, j): i<j \leq n\}| \leq 1$ for all $1 \leq i<n$, and if $|\mathcal{D} \cap\{(i, j): 1 \leq i<j\}| \leq 1$ for all $1<j \leq n$. In particular, the empty set is a basic subset of $\Phi$. Given an arbitrary nonempty basic subset $\mathcal{D}$ of $\Phi$ and given an arbitrary map $\varphi: \mathcal{D} \rightarrow \mathbb{F}_{q}^{\times}$, we define the basic character $\xi_{\mathcal{D}}(\varphi)$ of $U$ to be the product of elementary characters

$$
\xi_{\mathcal{D}}(\varphi)=\prod_{(i, j) \in \mathcal{D}} \xi_{i j}(\varphi(i, j)) .
$$

For our purposes, it is convenient to consider the trivial character $1_{U}$ of $U$ as the basic character $\xi_{\mathcal{D}}(\varphi)$ corresponding to the empty subset of $\Phi$ and to the empty function $\varphi: \mathcal{D} \rightarrow \mathbb{F}_{q}^{\times}$. By [2, Theorem 1], we know that every irreducible character $\chi$ of $U$ is a constituent of $\xi_{\mathcal{D}}(\varphi)$ for a unique basic subset $\mathcal{D} \subseteq \Phi$ and a unique map $\varphi: \mathcal{D} \rightarrow \mathbb{F}_{q}^{\times}$.

The purpose of this paper is to study the decomposition of an arbitrary basic character $\xi_{\mathcal{D}}(\varphi)$ of $U$. Throughout the paper, the basic subset $\mathcal{D} \subseteq \Phi$ and the map $\varphi: \mathcal{D} \rightarrow \mathbb{F}_{q}^{\times}$will be kept fixed. Moreover, we will simplify the notation and write $\xi$ to denote the basic character $\xi_{\mathcal{D}}(\varphi)$. We begin by proving that $\xi$ is induced from a linear character of a certain algebra subgroup of $U$. (Following [5], a subgroup of $U=1+\mathfrak{u}$ is called an algebra subgroup if it is of the form $1+J$ for some multiplicatively closed $\mathbb{F}_{q}$-subspace $J$ of $\mathfrak{u}$.) In order to construct this subgroup, we consider the $U$-action on $\mathfrak{u}^{*}$ given by left translation: for any $x \in U$ and any $f \in \mathfrak{u}^{*}$, we define the linear map $x f \in \mathfrak{u}^{*}$ by $(x f)(a)=f\left(x^{-1} a\right)$ for all $a \in \mathfrak{u}$. For any $f \in \mathfrak{u}^{*}$, let $U(f)=\{x \in U: x f=f\}$ be the centralizer of $f$ in $U$. Therefore, we have

$$
U(f)=\{x \in U: f(x b)=f(b) \text { for all } b \in \mathfrak{u}\} .
$$

On the other hand, let

$$
\mathfrak{u}(f)=\{a \in \mathfrak{u}: f(a b)=0 \text { for all } b \in \mathfrak{u}\} .
$$

It is easy to see that $\mathfrak{u}(f)$ is a multiplicatively closed $\mathbb{F}_{q}$-subspace of $\mathfrak{u}$. In fact, the following (easy) result holds. 
Lemma 1. For any $f \in \mathfrak{u}^{*}$, we have $U(f)=1+\mathfrak{u}(f)$; hence $U(f)$ is an algebra subgroup of $U$.

Proof. Let $a \in \mathfrak{u}(f)$ be arbitrary and let $x=1+a$. Then, $f(x b)=f(b+a b)=$ $f(b)+f(a b)=f(b)$ for all $b \in \mathfrak{u}$, and this implies that $x^{-1} \in U(f)$ (hence, $x \in U(f)$ ). Conversely, let $x \in U(f)$. Then, $f\left(x^{-1} b\right)=f(b)$ for all $b \in \mathfrak{u}$. Replacing $b$ by $x b$, we deduce that $f(b)=f(x b)$ for all $b \in \mathfrak{u}$, and so $a=x-1 \in \mathfrak{u}$ satisfies $f(a b)=0$ for all $b \in \mathfrak{u}$. This means that $a \in \mathfrak{u}(f)$, and so the equality $U(f)=1+\mathfrak{u}(f)$ holds.

As a special case, let $(i, j) \in \Phi$ and consider the element $e_{i j}^{*} \in \mathfrak{u}^{*}$. It is not difficult to show that $\mathfrak{u}\left(e_{i j}^{*}\right)=\left\{a \in \mathfrak{u}: a_{i k}=0\right.$ for all $\left.i<k<j\right\}$; hence,

$$
U\left(e_{i j}^{*}\right)=1+\mathfrak{u}\left(e_{i j}^{*}\right)=\left\{x \in U: x_{i k}=0 \text { for all } i<k<j\right\} .
$$

For simplicity, we write $\mathfrak{n}_{i j}=\mathfrak{u}\left(e_{i j}^{*}\right)$ and $N_{i j}=U\left(e_{i j}^{*}\right)$. Let $\alpha \in \mathbb{F}_{q}^{\times}$be arbitrary and let $\lambda_{i j}(\alpha): N_{i j} \rightarrow \mathbb{C}$ be defined by $\lambda_{i j}(\alpha)(x)=\psi\left(\alpha x_{i j}\right)$ for all $x \in U_{i j}(q)$. Then, by [2, Lemma 2], $\lambda_{i j}(\alpha)$ is a linear character of $N_{i j}$ and the $(i, j)$-th elementary character $\xi_{i j}(\alpha)$ is the induced character $\lambda_{i j}(\alpha)^{U}$. More generally, for the (arbitrarily) given basic subset $\mathcal{D}$ and for the map $\varphi: \mathcal{D} \rightarrow \mathbb{F}_{q}^{\times}$, let $e^{*} \in \mathfrak{u}^{*}$ denote the element

$$
e^{*}=\sum_{(i, j) \in \mathcal{D}} \varphi(i, j) e_{i j}^{*}
$$

and consider the centralizer $U\left(e^{*}\right)$ of $e^{*}$ in $U$. Moreover, let $\lambda: U\left(e^{*}\right) \rightarrow \mathbb{C}$ be the map defined by

$$
\lambda(1+a)=\psi\left(e^{*}(a)\right)
$$

for all $a \in \mathfrak{u}\left(e^{*}\right)$ (we recall that $U\left(e^{*}\right)=1+\mathfrak{u}\left(e^{*}\right)$ ). Then, $\lambda$ is a linear character of $U\left(e^{*}\right)$. In fact, let $x, y \in U\left(e^{*}\right)$ be arbitrary and let $a, b \in \mathfrak{u}\left(e^{*}\right)$ be such that $x=1+a$ and $y=1+b$. Then, $x y=1+a+b+a b$ and so

$$
\lambda(x y)=\lambda(x) \lambda(y) \psi\left(e^{*}(a b)\right)=\lambda(x) \lambda(y)
$$

(because $a, b \in \mathfrak{u}\left(e^{*}\right)$, hence $\left.e^{*}(a b)=0\right)$. We note that

$$
\lambda(x)=\prod_{(i, j) \in \mathcal{D}} \psi\left(\varphi(i, j) x_{i j}\right)
$$

for all $x \in U\left(e^{*}\right)$. In order to prove that $\xi=\lambda^{U}$, it is very useful to describe the centralizer $U\left(e^{*}\right)$ as follows. Let

$$
\mathcal{S}=\bigcup_{(i, j) \in \mathcal{D}}\{(i, k): i<k<j\} \subseteq \Phi,
$$

and let $\mathcal{R}=\Phi-\mathcal{S}$. Let $\mathfrak{n}$ be the $\mathbb{F}_{q}$-subspace of $\mathfrak{u}$ spanned by the vectors $e_{i j}$ for $(i, j) \in \mathcal{R}$. Then, $\mathfrak{n}=\left\{a \in \mathfrak{u}: a_{r s}=0\right.$ for all $\left.(r, s) \in \mathcal{S}\right\}$, and so $\mathfrak{n}=\bigcap_{(i, j) \in \mathcal{D}} \mathfrak{n}_{i j}$. In particular, we deduce that $\mathfrak{n}$ is a multiplicatively closed $\mathbb{F}_{q}$-subspace of $\mathfrak{u}$. Therefore, we may consider the algebra subroup $N=1+\mathfrak{n}$ of $U$. Then,

$$
N=\bigcap_{(i, j) \in \mathcal{D}} N_{i j}=\left\{x \in U: x_{r s}=0 \text { for all }(r, s) \in \mathcal{S}\right\} .
$$

We have the following result.

Lemma 2. The subgroup $N$ is the centralizer $U\left(e^{*}\right)$ of $e^{*}$ in $U$. 
Proof. We consider the standard basis $\left(e_{i j}:(i, j) \in \Phi\right)$ of $\mathfrak{u}$. By Lemma 1 it is enough to prove that $\mathfrak{n}$ consists of all matrices $a \in \mathfrak{u}$ that satisfy $e^{*}\left(a e_{i j}\right)=0$ for all $(i, j) \in \Phi$. Given an arbitrary element $a \in \mathfrak{u}$, we have $a e_{i j}=\sum_{1 \leq r<i} a_{r i} e_{r j}$. Therefore, $e^{*}\left(a e_{i j}\right)$ can be nonzero only if $(r, j) \in \mathcal{D}$ for some $1 \leq r<i$; and, if this is the case, we have $e^{*}\left(a e_{i j}\right)=a_{r i} \varphi(r, j)$. Now, let $a \in \mathfrak{n}$, let $(i, j) \in \Phi$ and suppose that $(r, j) \in \mathcal{D}$ for some $1 \leq r<i$. Then, $a_{r i}=0$ and so $e^{*}\left(a e_{i j}\right)=0$. It follows that $a \in \mathfrak{u}\left(e^{*}\right)$. Conversely, suppose that $a \in \mathfrak{u}\left(e^{*}\right)$ and let $(i, j) \in \mathcal{D}$. Then, for all $i<k<j$, we have $a_{i k} \varphi(i, j)=e^{*}\left(a e_{k j}\right)=0$ and so $a_{i k}=0$. Thus, $a \in \mathfrak{n}$ and the proof is complete.

We are now able to prove the following result.

Theorem 1. The basic character $\xi$ of $U$ is induced by the linear character $\lambda$ of $N$.

Proof. We proceed by induction on the cardinality $d$ of the set $\mathcal{D}$. The result is trivial if $d=0$ and, as we mentioned before, the case $d=1$ is given by [2] Lemma 2]. Now, suppose that $d>1$ and assume that the result is true for all the basic characters that correspond to the basic subsets $\mathcal{D}_{0} \subseteq \Phi$ with less than $d$ elements. Let $(i, j) \in \mathcal{D}$, let $\mathcal{D}_{0}=\mathcal{D}-\{(i, j)\}$, and let $\varphi_{0}: \mathcal{D}_{0} \rightarrow \mathbb{F}_{q}^{\times}$be the restriction of $\varphi$ to $\mathcal{D}_{0}$. Moreover, let $\alpha=\varphi(i, j)$, let $e_{0}^{*}=e^{*}-\alpha e_{i j}^{*}$, and let $N_{0}=U\left(e_{0}^{*}\right)$. Then, $N=N_{0} \cap N_{i j}$ and $U=N_{0} N_{i j}$. Let $\lambda_{0}: N_{0} \rightarrow \mathbb{C}$ be the linear character defined by $\lambda_{0}(1+a)=\psi\left(e_{0}^{*}(a)\right.$ ) for all $a \in \mathfrak{u}\left(e_{0}^{*}\right)$ (we recall that $\left.U\left(e_{0}^{*}\right)=1+\mathfrak{u}\left(e_{0}^{*}\right)\right)$. By induction, we know that $\left(\lambda_{0}\right)^{U}$ is the basic character $\xi_{0}=\xi_{\mathcal{D}_{0}}\left(\varphi_{0}\right)$ and so $\xi=\xi_{0} \zeta=\left(\lambda_{0}\right)^{U} \mu^{U}$ where $\zeta=\xi_{i j}(\alpha)$ and $\mu=\lambda_{i j}(\alpha)$. By Mackey's Subgroup Theorem (see [3, Theorem 10.13]), we have $\zeta_{N_{0}}=\left(\mu^{U}\right)_{N_{0}}=\left(\mu_{N}\right)^{N_{0}}$ and so

$$
\xi=\left(\lambda_{0}\right)^{U} \zeta=\left(\lambda_{0} \zeta_{N_{0}}\right)^{U}=\left(\lambda_{0}\left(\mu_{N}\right)^{N_{0}}\right)^{U} .
$$

Since $\lambda_{0}\left(\mu_{N}\right)^{N_{0}}=\left(\left(\lambda_{0}\right)_{N} \mu_{N}\right)^{N_{0}}$ and since $\lambda=\left(\lambda_{0}\right)_{N} \mu_{N}$ (as we observed above), we conclude that $\xi=\left(\lambda^{N_{0}}\right)^{U}=\lambda^{U}$.

Now, let $\mathbb{C}[U]$ (resp., $\mathbb{C}[N]$ ) be the group algebra of $U$ (resp., of $N$ ). As usual, we consider $\mathbb{C}[N]$ as a subalgebra of $\mathbb{C}[U]$. Let

$$
\varepsilon=\frac{1}{|N|} \sum_{x \in N} \overline{\lambda(x)} x \in \mathbb{C}[N]
$$

be the central primitive idempotent that corresponds to the linear character $\lambda$ of $N$ (hence, the left ideal $\mathbb{C}[N] \varepsilon$ of $\mathbb{C}[N]$ affords the character $\lambda$ of $N$; see [3] Proposition 9.21]). By [3, Proposition 11.21], the left ideal $\mathbb{C}[U] \varepsilon$ of $\mathbb{C}[U]$ affords the induced character $\lambda^{U}$ of $U$, and the multiplicity of an arbitrary irreducible character $\chi$ of $U$ as a constituent of $\lambda^{U}$ is given by the value $\chi(\varepsilon)$. Let $\mathcal{H}=\varepsilon \mathbb{C}[U] \varepsilon$ be the Hecke algebra associated with the linear character $\lambda$ of $N$. Since $\mathbb{C}[U]$ is a semisimple algebra, the Hecke algebra $\mathcal{H}$ is also semisimple (by Proposition 5.13 and Theorem 5.18 of [3]). Moreover, by [3, Theorem 11.25], the mapping $\chi \mapsto \chi_{\mathcal{H}}$ defines a bijection between the set of all irreducible constituents of $\lambda^{U}$ and the set of all irreducible characters of $\mathcal{H}$. In the following result, we describe a $\mathbb{C}$-basis of $\mathcal{H}$. First, we introduce some notation. Let $\mathcal{S}^{\prime} \subseteq \Phi$ be the subset consisting of all roots $(i, j) \in \Phi$ for which there exist $j<k<l \leq n$ with $(i, k),(j, l) \in \mathcal{D}$. It is clear that $\mathcal{S}^{\prime} \subseteq \mathcal{S}$. Let $\mathfrak{x}$ be the $\mathbb{F}_{q^{-}}$-subspace of $\mathfrak{u}$ spanned by the vectors $e_{i j}$ for $(i, j) \in \mathcal{S}^{\prime}$ and let $X=1+\mathfrak{x} \subseteq U$.

Proposition 1. For each $x \in U$, let ind $x=\left|N: N \cap x^{-1} N x\right|$ be the index of $x$ and let $a_{x}=($ ind $x) \varepsilon x \varepsilon \in \mathcal{H}$. Then, $\left(a_{x}: x \in X\right)$ is a $\mathbb{C}$-basis of $\mathcal{H}$. 
Proof. First, we observe that, for an arbitrary element $x \in U$, the intersection $x N x^{-1} \cap N$ is the algebra subgroup $1+\left(x \mathfrak{n} x^{-1} \cap \mathfrak{n}\right)$ of $U$. On the other hand, for any $b \in \mathfrak{n}$, we have $x b x^{-1} \in \mathfrak{n}$ if and only if $x b \in \mathfrak{n}$. In fact, since $\mathfrak{n}=\mathfrak{u}\left(e^{*}\right)$ (by Lemma 2), we have $x b x^{-1} \in \mathfrak{n}$ if and only if $e^{*}\left(x b x^{-1} a\right)=0$ for all $a \in \mathfrak{u}$. Replacing $a$ by $x a$, we conclude that $x b x^{-1} \in \mathfrak{n}$ if and only if $e^{*}(x b a)=0$ for all $a \in \mathfrak{u}$. It follows that $x b x^{-1} \in \mathfrak{n}$ if and only if $x b \in \mathfrak{u}\left(e^{*}\right)=\mathfrak{n}$, as required.

Next, we observe that each double coset of $N$ in $U$ may be represented by an element $x \in U$ with the form $x=1+a$ where $a \in \sum_{(i, j) \in \mathcal{S}} \mathbb{F}_{q} e_{i j}$. In fact, let us denote by $T$ the subset of $U$ consisting of all these elements. Then, $|T|=|U: N|$ and, in fact, $T$ is a complete set of representatives for the right cosets of $N$ in $U$ (hence, it contains a set of representatives for the double cosets of $N$ in $U$ ). To see this, let $x, y \in T$ be such that $x=z y$ for some $z \in N$. Moreover, let $a \in \mathfrak{n}$ be such that $z=1+a$, so that $x=y+a y$. Since $a \in \mathfrak{n}=\mathfrak{u}\left(e^{*}\right)$, we have $e^{*}(a b)=0$ for all $b \in \mathfrak{u}$. Therefore, we also have $e^{*}(a y b)=0$ for all $b \in \mathfrak{u}$, and so $a y \in \mathfrak{u}\left(e^{*}\right)=\mathfrak{n}$. It follows that $x-y=a y \in \mathfrak{n}$, and this clearly implies that $x=y$.

Now, by [3. Proposition 11.30], the Hecke algebra $\mathcal{H}$ has a $\mathbb{C}$-basis formed by some of the elements $a_{x}$ for $x \in T$ satisfying $\lambda\left(x^{-1} y x\right)=\lambda(y)$ for all $y \in x N x^{-1} \cap N$. We claim that such an element $x$ lies in $X$. Suppose that this is not the case. Then, there exists $(i, k) \in \Phi$ with $(i, k) \notin \mathcal{S}^{\prime}$ and $x_{i k} \neq 0$. Since $x \in T$, we must have $(i, k) \in \mathcal{S}$, and so there exists $k<j \leq n$ with $(i, j) \in \mathcal{D}$. By the definition of $\mathcal{S}^{\prime}$, we have $(k, l) \notin \mathcal{D}$ for all $j<l \leq n$. Moreover, we may choose the root $(i, j) \in \mathcal{D}$ such that, for any $(r, s) \in \mathcal{D}$ with $j<s \leq n$, we have $(r, t) \in \mathcal{S}^{\prime}$ whenever $r<t<s$ is such that $x_{r t} \neq 0$. Now, we claim that $x\left(1+e_{k j}\right) x^{-1} \in x N x^{-1} \cap N$ (we note that $1+e_{k j} \in N$, because $(k, j) \notin \mathcal{S}$ ). To prove this, it is enough to show that $x e_{k j} \in \mathfrak{n}$. In fact, let $(r, s) \in \mathcal{S}$ be arbitrary. Then, the $(r, s)$-th coefficient of $x e_{k j}$ can be nonzero only if $s=j$ and $r \leq k$; and, if this is the case, that coefficient is $x_{r k}$. Since $(r, j) \in \mathcal{S}$ (by our choice), there exists $j<t \leq n$ with $(r, t) \in \mathcal{D}$ and so, by the choice of $j$, we must have $x_{r k}=0$ (because $\left.(r, k) \notin \mathcal{S}^{\prime}\right)$. It follows that $x e_{k j} \in \mathfrak{n}$, as required. Now, let $\alpha \in \mathbb{F}_{q}$ be arbitrary and consider the $y_{\alpha}=x\left(1+\alpha e_{k j}\right) x^{-1}=1+\alpha x e_{k j} x^{-1}$. We note that $y_{\alpha} \in x N x^{-1} \cap N$ because $x e_{k j} x^{-1} \in \mathfrak{n}$ (hence $\alpha x e_{k j} x^{-1} \in \mathfrak{n}$ ). Therefore, by the definition of $T$, we have $\lambda\left(x^{-1} y_{\alpha} x\right)=\lambda\left(y_{\alpha}\right)$ and so

$$
\psi\left(\alpha e^{*}\left(e_{k j}\right)\right)=\psi\left(\alpha e^{*}\left(x e_{k j} x^{-1}\right)\right)
$$

(by the definition of $\lambda$ ). On the one hand, we have $e^{*}\left(e_{k j}\right)=0$ (because $(k, j) \notin \mathcal{D}$ ) and, on the other hand, we know that $x e_{k j} \in \mathfrak{n}$; hence $e^{*}\left(x e_{k j} b\right)=0$ for all $b \in \mathfrak{u}$ and this implies that $e^{*}\left(x e_{k j} z\right)=e^{*}\left(x e_{k j}\right)$ for all $z \in U$. In particular, we deduce that

$$
\psi\left(\alpha e^{*}\left(x e_{k j}\right)\right)=\psi\left(\alpha e^{*}\left(x e_{k j} x^{-1}\right)\right)=\psi\left(\alpha e^{*}\left(e_{k j}\right)\right)=1 .
$$

Now, suppose that $e^{*}\left(x e_{k j}\right) \neq 0$. Then, the mapping $\alpha \mapsto \alpha e^{*}\left(x e_{k j}\right)$ defines a permutation of $\mathbb{F}_{q}$, and so the equality $\psi\left(\alpha e^{*}\left(x e_{k j}\right)\right)=1$ (which holds for any $\alpha \in \mathbb{F}_{q}$ ) implies that $\psi$ is the trivial character of $\mathbb{F}_{q}^{+}$, contrary to the choice of $\psi$. Therefore, $e^{*}\left(x e_{k j}\right)=0$. However, $x e_{k j}=e_{k j}+\sum_{1 \leq r<k} x_{r k} e_{r j}$, and so

$$
e^{*}\left(x e_{k j}\right)=x_{i k} e^{*}\left(e_{i j}\right)=x_{i k} \varphi(i, j) \text {. }
$$

Since $\varphi(i, j) \neq 0$, we get a contradiction and so $x \in X$, as claimed.

By the result mentioned above, we conclude that the set $\left\{a_{x}: x \in X\right\}$ contains a $\mathbb{C}$-basis of $\mathcal{H}$. By the same result, to complete the proof we must show that, for $x, x^{\prime} \in X$ with $x \neq x^{\prime}$, the double cosets $N x N$ and $N x^{\prime} N$ are distinct. To see this, 
suppose that $x^{\prime}=y x z$ for some $y, z \in N$. Let $(i, j) \in \mathcal{S}^{\prime}$ be the largest root such that $x_{i j}^{\prime} \neq x_{i j}$; this means that $x_{r s}^{\prime}=x_{r s}$ whenever $(r, s) \in X$ is such that, either $j<s$, or $j=s$ and $r<i$. Since $(i, j) \in \mathcal{S}$ and since $y \in N$, we have $y_{i r}=0$ for all $i<r<j$, and so $x_{i j}^{\prime}=\sum_{i<s<j} x_{i s} z_{s j}$. Now, suppose that $x_{i s} \neq 0$ for some $i<s<j$. Then, $(i, s) \in \mathcal{S}^{\prime}$ and so there exists $j+1<k \leq n$ with $(s, k) \in \mathcal{D}$. Therefore, $(s, j) \in \mathcal{S}$ and this implies that $z_{s j}=0$ (because $z \in N$ ). It follows that $x_{i j}^{\prime}=x_{i j}+z_{i j}$. However, $(i, j) \in \mathcal{S}$; hence $z_{i j}=0$. Therefore, $x_{i j}^{\prime}=x_{i j}$ and this contradiction implies that the double cosets $N x N$ and $N x^{\prime} N$ are distinct.

In the next result, we show that the $\mathbb{C}$-basis $\left(a_{x}: x \in X\right)$ is (in certain sense) a "group basis" of $\mathcal{H}$.

Proposition 2. The $\mathbb{F}_{q}$-subspace $\mathfrak{s}=\mathfrak{n} \oplus \mathfrak{x}$ of $\mathfrak{u}$ is multiplicatively closed, and so $S=1+\mathfrak{s}$ is an algebra subgroup of $U$. Moreover, $N$ is a normal subgroup of $S$ and $X$ is a complete set of representatives of the elements of the quotient group $S / N$.

Proof. We recall that $\mathfrak{n}$ is spanned by the vectors $e_{i j}$ for $(i, j) \in \mathcal{R}$ where $\mathcal{R}=\Phi-\mathcal{S}$. Therefore, the first assertion of the proposition will follow once we prove that the (disjoint) union $\mathcal{R} \cup \mathcal{S}^{\prime}$ is a closed subset of $\Phi$, i.e., we have $(i, k) \in \mathcal{R} \cup \mathcal{S}^{\prime}$ whenever $(i, j),(j, k) \in \mathcal{R} \cup \mathcal{S}^{\prime}$. This is clear in the case where $(i, j),(j, k) \in \mathcal{R}$. It is also clear that $(i, k) \in \mathcal{R}$ in the case where $(i, j) \in \mathcal{R}$ and $(j, k) \in \mathcal{S}^{\prime}$. Now, suppose that $(i, j) \in \mathcal{S}^{\prime}$ and that $(j, k) \in \mathcal{R}$. By definition of $\mathcal{S}^{\prime}$, there exist $(i, r),(j, s) \in$ $\mathcal{D}$ with $j<r$. Moreover, we must have $s \leq k$ because $(j, k) \in \mathcal{R}$. Therefore, $r<k$ and so $(i, k) \in \mathcal{R}$. Finally, suppose that $(i, j),(j, k) \in \mathcal{S}^{\prime}$. Then, there exist $(i, r),(j, s),(k, t) \in \mathcal{D}$ with $j<r$ and $k<s$. We have two cases: on the one hand, if $r \leq k$, then $(i, k) \in \mathcal{R}$; on the other hand, if $k<r$, then $(i, k) \in \mathcal{S}^{\prime}$ because $(i, r),(k, t) \in \mathcal{D}$.

For the second assertion, we note that, since $N=1+\mathfrak{n}$ and since $x(1+a) x^{-1}=$ $1+\operatorname{xax}^{-1}$ for all $x \in U$ and all $a \in \mathfrak{u}$, it is enough to prove that $\operatorname{xax}^{-1} \in \mathfrak{n}$ for all $x \in S$ and all $a \in \mathfrak{n}$. Let $x \in S$ and let $a \in \mathfrak{n}$ be arbitrary. Then, by Lemma 2 we have $x a x^{-1} \in \mathfrak{n}$ if and only if $e^{*}\left(x a x^{-1} b\right)=0$ for all $b \in \mathfrak{u}$. Replacing $b$ by $x b$, we conclude that $x a x^{-1} \in \mathfrak{n}$ if and only if $e^{*}(x a b)=0$ for all $b \in \mathfrak{u}$. Therefore, we have $x a x^{-1} \in \mathfrak{n}$ if and only if $x a \in \mathfrak{n}$. Now, let $(r, s) \in \mathcal{S}$. Then, $(x a)_{r s}=\sum_{r \leq t \leq s} x_{r t} a_{t s}$. If $a_{t s} \neq 0$, we must have $(t, s) \in \mathcal{R}$. On the other hand, $x_{r t}$ can be nonzero only if $(r, t) \in \mathcal{S}^{\prime}$. Therefore, there exist $(r, u),(t, v) \in \mathcal{D}$ with $u<v$. Since $(r, s) \in \mathcal{S}$, we must have $s<u<v$ and so $(t, s) \in \mathcal{S}$. This contradiction implies that $(r, t) \notin \mathcal{S}^{\prime}$ and so $x_{r t}=0$. It follows that $(x a)_{r s}=0$, and this implies that $x a \in \mathfrak{n}$.

Corollary 1. For any $x \in X$, we have $a_{x}=\varepsilon x=x \varepsilon$. In particular, $(x \varepsilon: x \in X)$ is a $\mathbb{C}$-basis of $\mathcal{H}$.

Proof. Let $x \in X$ be arbitrary. Then, $x \in S$ and so ind $x=1$ (because $N$ is normal in $S$ ). Moreover, since $\lambda$ is $S$-invariant (by definition of $X$ ), we deduce that

$$
x \varepsilon=\frac{1}{|N|} \sum_{y \in N} \lambda\left(y^{-1}\right) x y=\frac{1}{|N|} \sum_{z \in N} \lambda\left(x^{-1} z^{-1} x\right) z x=\varepsilon x .
$$

The result follows because $\varepsilon$ is an idempotent.

Now, let $\mathbb{C}[S]$ be the group algebra of $S$. Then, $\mathbb{C}[S]$ is a subalgebra of $\mathbb{C}[U]$ and so $\mathcal{H}_{0}=\varepsilon \mathbb{C}[S] \varepsilon$ is a subalgebra of $\mathcal{H}$. Since $a_{x} \in \mathcal{H}_{0}$ for all $x \in X$, we conclude that $\mathcal{H}_{0}=\mathcal{H}$. Since $\mathcal{H}_{0}$ is the Hecke algebra associated with the (normal) subgroup 
$N$ of $S$ and with the linear character $\lambda$ of $N$, we may use [3, Theorem 11.25] to deduce the following result.

Theorem 2. The mapping $\phi \mapsto \phi^{U}$ defines a bijection between the set of all irreducible constituents of the induced character $\lambda^{S}$ and the set of all irreducible constituents of the basic character $\xi$. Moreover, this bijection preserves multiplicities, i.e., $\left\langle\phi^{U}, \xi\right\rangle_{U}=\left\langle\phi, \lambda^{S}\right\rangle_{S}$ for all irreducible constituents $\phi$ of $\lambda^{S}$. (Given any finite group $G$, we denote by $\langle\cdot, \cdot\rangle_{G}$ the usual Frobenius scalar product on the $\mathbb{C}$-space of all class functions of $G$.)

Proof. By [3, Theorem 11.25], the mapping $\chi \mapsto \chi_{\mathcal{H}}$ defines a bijection between the set of all irreducible constituents of $\lambda^{U}$ and the set of all irreducible characters of $\mathcal{H}$. By the same result (and by the paragraph above), the mapping $\phi \mapsto \phi_{\mathcal{H}}$ defines a bijection between the set of irreducible constituents of $\lambda^{S}$ and the set of irreducible characters of $\mathcal{H}$. Therefore, the irreducible constituents of $\lambda^{U}$ are in one-to-one correspondence with the irreducible constituents of $\lambda^{S}$.

Now, let $\chi \in \operatorname{Irr}(U)$ be a constituent of $\lambda^{U}$ and let $\theta=\chi_{\mathcal{H}}$ be the irreducible character of $\mathcal{H}$ that corresponds to $\chi$. (Given any finite group $G$, we denote by $\operatorname{Irr}(G)$ the set of all irreducible characters of $G$.) On the other hand, let $\phi \in \operatorname{Irr}(S)$ be the (unique) constituent of $\lambda^{S}$ such that $\phi_{\mathcal{H}}=\theta$. We claim that $\chi=\phi^{U}$. To see this, let $\chi^{\prime} \in \operatorname{Irr}(U)$ be any irreducible constituent of $\phi^{U}$. Then, $\chi^{\prime}$ is a constituent of $\lambda^{U}$ and so $\chi_{\mathcal{H}}^{\prime}$ is an irreducible character of $\mathcal{H}$. Since $\phi$ is a constituent of $\chi_{S}^{\prime}$ (by Frobenius reciprocity), we conclude that $\theta$ is a constituent of $\chi_{\mathcal{H}}^{\prime}$ and so $\theta=\chi_{\mathcal{H}}^{\prime}$ (because $\theta$ and $\chi_{\mathcal{H}}^{\prime}$ are irreducible). Therefore, $\chi^{\prime}$ is the unique irreducible constituent of $\lambda^{U}$ with $\chi_{\mathcal{H}}^{\prime}=\theta$. Thus, $\chi^{\prime}=\chi$ and so $\chi$ is the unique irreducible constituent of $\phi^{U}$. It follows that $\phi^{U}=m \chi$ where $m=\left\langle\phi^{U}, \chi\right\rangle_{U}$. In particular, we have $\phi^{U}(1)=m \chi(1)$ and so $|U: S| \phi(1)=m \chi(1)$. Finally, for each $x \in X$, let $\widehat{a}_{x}=\varepsilon x^{-1} \varepsilon \in \mathcal{H}$. Then, by [3, Theorem 11.32], we have

$$
c \chi(1)=|U: N|\left\langle\chi, \lambda^{U}\right\rangle_{U}
$$

where $c=\sum_{x \in X} \theta\left(\widehat{a}_{x}\right) \theta\left(a_{x}\right)$ (we recall that ind $x=1$ for all $x \in X$ ). Similarly,

$$
c \phi(1)=|S: N|\left\langle\phi, \lambda^{S}\right\rangle_{S} .
$$

Since $\left\langle\chi, \lambda^{U}\right\rangle_{U}=\theta(\varepsilon)=\left\langle\phi, \lambda^{S}\right\rangle_{S}$ (by [3, Theorem 11.25]), we deduce that

$$
\chi(1)=|U: S| \phi(1)=m \chi(1)
$$

and so $m=1$. It follows that $\phi^{U}=\chi$ is an irreducible constituent of $\lambda^{U}=\xi$.

Next, we identify a distinguished irreducible constituent of the arbitrary basic character $\xi$ of $U$. In particular, we generalize 1, Corollary 5] for an arbitary prime, proving that $\xi$ has a unique irreducible constituent if and only if the derived set $\mathcal{D}^{\prime}$ of $\mathcal{D}$ is empty. We start by recalling the definition of $\mathcal{D}^{\prime}$. A chain in $\Phi$ (of length $r-1)$ is a subset $\mathcal{C} \subseteq \Phi$ with the form $\mathcal{C}=\left\{\left(i_{1}, i_{2}\right),\left(i_{2}, i_{3}\right), \ldots,\left(i_{r-1}, i_{r}\right)\right\}$. Given two chains $\mathcal{C}_{1}=\left\{\left(i_{1}, i_{2}\right),\left(i_{2}, i_{3}\right), \ldots,\left(i_{r-1}, i_{r}\right)\right\}$ and $\mathcal{C}_{2}=\left\{\left(j_{1}, j_{2}\right),\left(j_{2}, j_{3}\right), \ldots,\left(j_{s-1}, j_{s}\right)\right\}$ in $\Phi$, we say that $\mathcal{C}_{1}$ and $\mathcal{C}_{2}$ intertwine if $r=s$ and if $i_{t}<j_{t}<i_{t+1}<j_{t+1}$ for all $1 \leq t \leq r-1$. Finally, given a basic subset $\mathcal{D} \subseteq \Phi$, we say that a root $(i, j) \in \Phi$ is $\mathcal{D}$-derived if there exist two intertwining chains $\mathcal{C}_{1}, \mathcal{C}_{2} \subseteq \mathcal{D}$ (of length $r-1$ ) with $i=i_{1}$ and $j=j_{1}$ (where the notation is as above) satisfying the following two conditions: (1) if $\left(i_{0}, i_{1}\right) \in \mathcal{D}$ for some $1 \leq i_{0}<i_{1}$, then $j_{1}<i_{0} ;(2)$ if $\left(j_{r}, j_{r+1}\right) \in \mathcal{D}$ for some $j_{r}<j_{r+1} \leq n$, then $j_{r+1}<i_{r}$. We denote by $\mathcal{D}^{\prime}$ the set of all $\mathcal{D}$-derived roots and call it the derived set of $\mathcal{D}$. Now, the set $\mathcal{S}^{\prime}$ can be decomposed as 
a disjoint union of maximal chains. Let $\mathcal{C}=\left\{\left(i_{1}, i_{2}\right),\left(i_{2}, i_{3}\right), \ldots,\left(i_{r}, i_{r+1}\right)\right\} \subseteq \mathcal{S}^{\prime}$ be a chain. Then, by the definition of $\mathcal{S}^{\prime}$, the sets $\mathcal{C}_{1}=\left\{\left(i_{1}, i_{3}\right),\left(i_{3}, i_{5}\right), \ldots\right\}$ and $\mathfrak{C}_{2}=\left\{\left(i_{2}, i_{4}\right),\left(i_{4}, i_{6}\right), \ldots\right\}$ are intertwinning chains in $\mathcal{D}$. On the other hand, it is clear that the chain $\mathcal{C}$ is maximal in $\mathcal{S}^{\prime}$ if and only if $\mathcal{S}^{\prime}$ does not contain roots $\left(i, i_{1}\right),\left(i_{r+1}, j\right) \in \Phi$ (for some $1 \leq i<i_{1}$ and some $\left.i_{r+1}<j \leq n\right)$. We note that, if $\mathcal{C}$ is maximal in $\mathcal{S}^{\prime}$, the root $\left(i_{1}, i_{2}\right) \in \mathcal{C}$ is $\mathcal{D}$-derived if and only if the length $r$ of $\mathcal{C}$ is odd. Moreover, every $\mathcal{D}$-derived root (if it exists) must appear as the initial root of a unique maximal chain in $\mathcal{S}^{\prime}$. Hence, the derived set $\mathcal{D}^{\prime}$ is empty if and only if all maximal chains in $\mathcal{S}^{\prime}$ have even length.

Now, we define the subset $\mathcal{S}_{0}^{\prime}$ of $\mathcal{S}^{\prime}$ as follows. Let $(i, j) \in \mathcal{S}^{\prime}$ be arbitrary and let $\mathcal{C}=\left\{\left(i_{1}, i_{2}\right),\left(i_{2}, i_{3}\right), \ldots,\left(i_{r}, i_{r+1}\right)\right\}$ be the maximal chain in $\mathcal{S}^{\prime}$ that contains the root $(i, j)$. Let $1 \leq s \leq r$ be such that $(i, j)=\left(i_{s}, i_{s+1}\right)$. Then, $(i, j) \in \mathcal{S}_{0}^{\prime}$ if and only if the subchain $\mathcal{C}_{i j}=\left\{\left(i_{s}, i_{s+1}\right), \ldots,\left(i_{r}, i_{r+1}\right)\right\}$ of $\mathcal{C}$ has odd length. Therefore, we have $\mathcal{S}_{0}^{\prime} \cap \mathcal{C}=\left\{\left(i_{r}, i_{r+1}\right),\left(i_{r-2}, i_{r-1}\right), \ldots\right\}$. We have the following result.

Lemma 3. Let $\mathfrak{x}_{0}$ be the $\mathbb{F}_{q}$-subspace of $\mathfrak{u}$ spanned by the vectors $e_{i j}$ for $(i, j) \in \mathcal{S}_{0}^{\prime}$. Then, the $\mathbb{F}_{q}$-subspace $\mathfrak{p}=\mathfrak{n} \oplus \mathfrak{x}_{0}$ of $\mathfrak{u}$ is multiplicatively closed and so $P=1+\mathfrak{p}$ is an algebra subgroup of $S$.

Proof. Suppose that $\mathfrak{p}$ is not multiplicatively closed. Then, we may choose the largest $1 \leq k \leq n$ with the property that there exist $(i, j),(j, k) \in \mathcal{R} \cup \mathcal{S}_{0}^{\prime}$ with $(i, k) \notin \mathcal{R} \cup \mathcal{S}_{0}^{\prime}$ (we recall that $\mathcal{R}=\Phi-\mathcal{S}$ ). If $(i, j) \in \mathcal{R}$, then $(i, k) \in \mathcal{R}$. Therefore, we must have $(i, j) \in \mathcal{S}_{0}^{\prime}$; hence $(i, j) \in \mathcal{S}^{\prime}$ and so there exist $(i, r),(j, s) \in \mathcal{D}$ with $r<s$. If $(j, k) \in \mathcal{R}$, then $s \leq k$ and so $(i, k) \in \mathcal{R}$ (because $r<s \leq k$ and $(i, r) \in \mathcal{D})$. Since this cannot happen, we must have $(j, k) \in \mathcal{S}_{0}^{\prime}$ and so $k<s$ (because $\mathcal{S}_{0}^{\prime} \subseteq \mathcal{S}^{\prime} \subseteq \mathcal{S}$ ). If $r \leq k$, then $(i, k) \in \mathcal{R}$, which cannot happen. Therefore, we have $k<r$. Since $(j, k) \in \mathcal{S}_{0}^{\prime} \subseteq \mathcal{S}^{\prime}$, there exists $(k, t) \in \mathcal{D}$ with $s<t$. Now, since $(i, k) \notin \mathcal{S}_{0}^{\prime}$, there exists $(r, u) \in \mathcal{D}$ with $t<u$ (otherwise $(r, t) \in \mathcal{R}$ and this implies that $\left.(i, k) \in \mathcal{S}_{0}^{\prime}\right)$. It follows that $(k, r) \in \mathcal{S}^{\prime}$ and so $(k, r) \in \mathcal{S}_{0}^{\prime}$ (otherwise, $\left.(i, k) \in \mathcal{S}_{0}^{\prime}\right)$. Hence, we have $(j, k),(k, r) \in \mathcal{S}_{0}^{\prime}$. Since $(j, s),(r, u) \in \mathcal{D}$ and since $s<u$, we have $(j, r) \in \mathcal{S}^{\prime}$. On the other hand, since $(i, j) \in \mathcal{S}_{0}^{\prime}$, the root $(j, r)$ does not lie in $\mathcal{S}_{0}^{\prime}$. Since $k<r$, this contradicts the choice of $k$. The proof is complete.

Let $\mu: P \rightarrow \mathbb{C}$ be the map defined by

$$
\mu(1+a)=\psi\left(e^{*}(a)\right)
$$

for all $a \in \mathfrak{p}$. We claim that $\mu$ is a linear character of $P$. To see this, let $a, b \in \mathfrak{p}$ be arbitrary and let $x=1+a$ and $y=1+b$. Then, $x y=1+a+b+a b$ and so $\mu(x y)=\mu(x) \mu(y) \psi\left(e^{*}(a b)\right)$. Since $e^{*}\left(e_{i k}\right) \neq 0$ if and only if $(i, k) \in \mathcal{D}$, we clearly have $e^{*}\left(e_{i j} e_{j k}\right)=e^{*}\left(e_{i k}\right)=0$ for all $(i, j),(j, k) \in \mathcal{R} \cup \mathcal{S}_{0}^{\prime}$. It follows that $e^{*}(a b)=0$ and so $\mu(x y)=\mu(x) \mu(y)$. Therefore, $\mu$ is in fact a linear character of $P$. (Moreover, it is clear that $\mu_{N}=\lambda$ and so, by Gallagher's Theorem (see [3, Theorem 11.5]), we have $\lambda^{P}=\sum_{\omega} \omega(1)(\omega \mu)$ where the sum extends over all the irreducible characters of the quotient group $P / N$ (viewed as characters of $P$ ).) In the following, we prove that the induced character $\mu^{U}$ is irreducible and that, in fact, $\mu^{U}=\phi_{\mathcal{O}}$ where $\mathcal{O} \subseteq \mathfrak{u}^{*}$ is the coadjoint $U$-orbit that contains the element $e^{*}$. We start by proving the following result.

Proposition 3. The induced character $\mu^{S}$ is an irreducible constituent of $\lambda^{S}$. Moreover, let $\mathcal{O} \subseteq \mathfrak{s}^{*}$ be the coadjoint $S$-orbit that contains the element $e^{*} \in \mathfrak{s}^{*}$. 
Then, we have

$$
\mu^{S}(1+a)=\frac{1}{\sqrt{|\mathcal{O}|}} \sum_{f \in \mathcal{O}} \psi(f(a))
$$

for all $a \in \mathfrak{s}$.

Proof. By [2 Proposition 1]), the induced character $\mu^{S}$ is a linear combination of the class functions $\phi_{\mathcal{O}^{\prime}}$ corresponding to the coadjoint $S$-orbits $\mathcal{O}^{\prime} \subseteq \mathfrak{s}^{*}$. Let $\pi: \mathfrak{s}^{*} \rightarrow \mathfrak{p}^{*}$ be the natural projection (given by the restriction of functions) and let $\Omega^{*}$ denote the set of all coadjoint $S$-orbits $\mathcal{O}^{\prime} \subseteq \mathfrak{s}^{*}$ such that $e^{*} \in \pi\left(\mathcal{O}^{\prime}\right)$ (here, we abuse the notation and write $e^{*}$ instead of $\left.\pi\left(e^{*}\right)\right)$. Since $e^{*}([a, b])=0$ for all $a, b \in \mathfrak{p}$, the group $P$ centralizes the element $e^{*} \in \mathfrak{p}^{*}$ and so $\left\{e^{*}\right\}$ is a single coadjoint $P$-orbit on $\mathfrak{p}^{*}$. Therefore, by [2, Proposition 2] (and by Frobenius reciprocity), $\mu^{S}$ is a linear combination of the class functions $\phi_{\mathcal{O}^{\prime}}$ for $\mathcal{O}^{\prime} \in \Omega^{*}$; moreover, for each $\mathcal{O}^{\prime} \in \Omega^{*}$, the multiplicity $m_{\mathcal{O}^{\prime}}=\left\langle\mu^{S}, \phi_{\mathcal{O}^{\prime}}\right\rangle_{S}$ is a positive integer.

Now, let $B: \mathfrak{s} \times \mathfrak{s} \rightarrow \mathbb{F}_{q}$ be the skew-symmetric $\mathbb{F}_{q}$-bilinear form defined by $B(a, b)=f([a, b])$ for all $a, b \in \mathfrak{s}$ and let $\mathfrak{r}=\left\{a \in \mathfrak{s}: e^{*}([a, b])=0\right.$ for all $\left.b \in \mathfrak{s}\right\}$ be the radical of $B$. It is well known that $m=\operatorname{dim} \mathfrak{s}-\operatorname{dim} \mathfrak{r}$ is an even number. Moreover, by [2 Lemma 1], we have $|\mathcal{O}|=q^{m}$. On the other hand, let $M$ be the matrix with entries $e^{*}\left(\left[e_{i j}, e_{k l}\right]\right)$ for $(i, j),(k, l) \in \mathcal{S}^{\prime} \cup \mathcal{R}$. Then, since $\operatorname{rank} M=$ $\operatorname{dim} \mathfrak{s}-\operatorname{dim} \mathfrak{r}$, we have $|\mathcal{O}|=q^{\text {rank } M}$. Let $\mathfrak{C}_{1}, \ldots, \mathcal{C}_{t}$ be the distinct maximal chains in $\mathcal{S}^{\prime}$ and, for each $1 \leq s \leq t$, let $M_{s}$ be the submatrix of $M$ defined by the roots $(i, j) \in \mathcal{C}_{s}$. It is easy to see that $\operatorname{rank} M=\operatorname{rank} M_{1}+\cdots+\operatorname{rank} M_{t}$ and that, for each $1 \leq s \leq t$,

$$
\operatorname{rank} M_{s}= \begin{cases}\left|\mathcal{C}_{s}\right|, & \text { if }\left|\mathfrak{C}_{s}\right| \text { is even, } \\ \left|\mathcal{C}_{s}\right|-1, & \text { if }\left|\mathfrak{C}_{s}\right| \text { is odd }\end{cases}
$$

(see the proof of [1, Theorem 3]). It follows that $\operatorname{rank} M=\left|\mathcal{S}^{\prime}\right|-\left|\mathcal{D}^{\prime}\right|$. Since $\operatorname{dim} \mathfrak{s}-\operatorname{dim} \mathfrak{p}=\frac{1}{2}\left(\left|\mathcal{S}^{\prime}\right|-\left|\mathcal{D}^{\prime}\right|\right)$, we conclude that $|\mathcal{O}|=q^{\operatorname{rank} M}=q^{2(\operatorname{dim} \mathfrak{s}-\operatorname{dim} \mathfrak{p})}=$ $|S: P|^{2}$. Finally, since

$$
|S: P|=\mu^{S}(1)=\sum_{\mathcal{O}^{\prime} \in \Omega^{*}} m_{\mathcal{O}^{\prime}} \phi_{\mathcal{O}^{\prime}}(1)=\sum_{\mathcal{O}^{\prime} \in \Omega^{*}} m_{\mathcal{O}^{\prime}} \sqrt{\left|\mathcal{O}^{\prime}\right|}
$$

(and since $\mathcal{O} \in \Omega^{*}$ ), we conclude that $\Omega^{*}=\{\mathcal{O}\}$, that $m_{\mathcal{O}}=1$ and that $\mu^{S}=\phi_{\mathcal{O}}$. Since $\left\langle\phi_{\mathcal{O}}, \phi_{\mathcal{O}}\right\rangle_{S}=1$ (by [2, Proposition 1]), the induced character $\mu^{S}$ is irreducible. Moreover, $\mu^{S}$ is a constituent of $\lambda^{S}$ because $\mu$ is a constituent of $\lambda^{P}$.

We now may apply Theorem 2 to justify the first assertion of the following corollary.

Theorem 3. The induced character $\mu^{U}$ is an irreducible constituent of $\xi$. Moreover, $\mu^{U}$ is the class function $\phi_{\mathcal{O}}$ that corresponds to the coadjoint $U$-orbit $\mathcal{O} \subseteq \mathfrak{u}^{*}$ that contains the element $e^{*} \in \mathfrak{u}^{*}$.

Proof. It remains to show that $\mu^{U}=\phi_{\mathcal{O}}$. Let $\mathcal{O}_{0} \subseteq \mathfrak{s}^{*}$ be the coadjoint $S$-orbit that ontains the element $e^{*} \in \mathfrak{s}^{*}$. By the previous proposition, we have $\mu^{S}=\phi_{\mathcal{O}_{0}}$ and so $\mu^{U}=\left(\phi_{\mathcal{O}_{0}}\right)^{U}$. Therefore, we must prove that $\left(\phi_{\mathcal{O}_{0}}\right)^{U}=\phi_{\mathcal{O}}$. Let $\pi: \mathfrak{u}^{*} \rightarrow \mathfrak{s}^{*}$ be the natural projection. Since $\mathcal{O}_{0} \subseteq \pi(\mathcal{O})$, the class function $\phi_{\mathcal{O}}$ occurs as a constituent of $\left(\phi_{\mathcal{O}_{0}}\right)^{U}=\mu^{U}$ with positive integer multiplicity (by [2] Proposition 2] and by Frobenius reciprocity). Let $M$ be the matrix with entries $e^{*}\left(\left[e_{i j}, e_{k l}\right]\right)$ for $(i, j),(k, l) \in \Phi$. It is easy to prove that $\operatorname{rank} M=\operatorname{rank} M_{0}+2\left(|\mathcal{S}|-\left|\mathcal{S}^{\prime}\right|\right)$ where $M_{0}$ 
is the submatrix of $M$ defined by the roots $(i, j) \in \mathcal{R} \cup \mathcal{S}^{\prime}$. As in the proof of the previous proposition, we conclude that

$$
|\mathcal{O}|=q^{\operatorname{rank} M}=q^{\operatorname{rank} M_{0}}=q^{2\left(|\mathcal{S}|-\left|\mathcal{S}^{\prime}\right|\right)}=\left|\mathcal{O}_{0}\right||U: S|^{2}
$$

and so

$$
\phi_{\mathcal{O}}(1)=\sqrt{|\mathcal{O}|}=|U: S| \sqrt{\left|\mathcal{O}_{0}\right|}=|U: S| \phi_{\mathcal{O}_{0}}(1)=\left(\phi_{\mathcal{O}_{0}}\right)^{U}(1) .
$$

It follows that $\phi_{\mathcal{O}}=\left(\phi_{\mathcal{O}_{0}}\right)^{U}$, and this completes the proof.

Finally, 2, Theorems 1 and 2] imply that

$$
\left\langle\mu^{U}, \xi\right\rangle_{U}=q^{s^{\prime}-s} \mu^{U}(1)
$$

where $s=|\mathcal{S}|$ and $s^{\prime}=\left|\mathcal{S}^{\prime}\right|$; we note that, since $\xi=\lambda^{U}$ (by Theorem 1), we have $\left.\xi(1)=|U: N|=q^{s}\right)$. On the other hand, it is easy to see that $q^{s^{\prime}}=|S: N|$. Since $\mu^{U}(1)=|U: P|$, we conclude that $\left\langle\mu^{U}, \xi\right\rangle_{U}=|S: P|$. Now, suppose that the derived set $\mathcal{D}^{\prime}$ of $\mathcal{D}$ is empty. Then, all maximal chains in $\mathcal{S}^{\prime}$ have even length and so $|S: P|=\sqrt{q^{s^{\prime}}}=\mu^{S}(1)$ (see the proof of Proposition 3). On the other hand, by Theorem 2, we know that

$$
\left\langle\mu^{S}, \lambda^{S}\right\rangle_{S}=\left\langle\left(\mu^{S}\right)^{U}, \xi\right\rangle_{U}=\left\langle\mu^{U}, \xi\right\rangle_{U}=|S: P| \text {. }
$$

Since $\lambda^{S}(1)=|S: N|$ and since $|S: N|=|S: P|^{2}$ (because $\mathcal{D}^{\prime}=\emptyset$ ), we conclude that $\lambda^{S}=|S: P| \mu^{S}$. Therefore, we deduce the following result (see [1, Corollary 5] for the case where $p \geq n$ ).

Theorem 4. The basic character $\xi$ has a unique irreducible constituent if and only if the derived set $\mathcal{D}^{\prime}$ of $\mathcal{D}$ is empty. If this is the case, we have $\xi=m \mu^{U}$ where $m=|S: P|$ (and $\left.m^{2}=|S: N|\right)$.

Proof. If $\mathcal{D}^{\prime}$ is empty, we deduce that $\xi=\lambda^{U}=\left(\lambda^{S}\right)^{U}=m\left(\mu^{S}\right)^{U}=m \mu^{U}$. In the general situation, we know that $\mu^{S}$ is an irreducible constituent of $\lambda^{S}$ with multiplicity $m=|S: P|$. On the other hand, it is easy to see that $|P: N|=$ $q^{\left|\mathcal{D}^{\prime}\right|}|S: P|$ and so $|S: N|=q^{\left|\mathcal{D}^{\prime}\right|}|S: P|^{2}$. Therefore, $\lambda^{S}(1)=m q^{\left|\mathcal{D}^{\prime}\right|} \mu^{S}(1)$ and this implies that $\lambda^{S}=m \mu^{S}$ if and only if $\mathcal{D}^{\prime}$ is empty. The result follows (using Theorem [2).

\section{REFERENCES}

[1] C. A. M. André, Basic sums of coadjoint orbits of the unitriangular group, J. Algebra 176 (1995), 959-1000. MR 96h:20081b

[2] C. A. M. André, Basic characters of the unitriangular group (for arbitrary primes), Proc. Amer. Math. Soc. 130, no. 7, (2002), 1943-1954.

[3] C. W. Curtis and I. Reiner, Methods of representation theory (with applications to finite groups and orders), Vol. 1, Wiley-Interscience, New York, 1981. MR 82i:20001

[4] B. Huppert, Character theory of finite groups, Walter de Gruyter, Berlin, 1998. MR 99j:20011

[5] I. M. Isaacs, Characters of groups associated with finite algebras, J. Algebra 177 (1995) 708730. MR 96k:20011

[6] I. M. Isaacs and D. Karagueuzian, Conjugacy in groups of upper triangular matrices, J. Algebra 202 (1998), 704-711. MR 99b:20011

Departamento de Matemática e Centro de Estruturas lineares e Combinatórias, Faculdade de Ciências da Universidade de Lisboa, Rua Ernesto de Vasconcelos, Edifício C1, Piso 3, 1749-016 Lisboa, Portugal

E-mail address: candre@f.ul.pt 